\title{
INFINITE LINEAR WAVEGUIDE ANTENNA ARRAY WITH METAL- DIELECTRIC STRUCTURES IN THE "FLOQUET CHANNEL"
}

\author{
V.M. Morozov, V.I. Magro*, E.Yu. Trakhtman \\ Oles Honchar Dnipro National University, Dnipro, Ukraine \\ *e-mail:magrov@i.ua
}

\begin{abstract}
An electrodynamics calculation is performed for an infinite waveguide antenna array in the form of open ends of waveguides, in the near zone of which a metal-dielectric structure is located. This metaldielectric structure is located in the "Floquet channels" and is a combination of a dielectric coating over the antenna array and horns. In this case, the horns are not a continuation of the waveguides, but are located at some distance from them. The space between the waveguides and the horns is filled with a dielectric. A system of equations that allows calculating the reflection coefficients of incident waves in waveguides is obtained.
\end{abstract}

Keywords: waveguide, antenna array, horn, integral equation, method, reflection coefficient.

Received 05.07.2020; Received in revised form 20.08.2020; Accepted 04.09.2020

\section{Introduction}

Metal dielectric structures can be used in a variety of microwave devices. Such metaldielectric structures are used in microstrip lines [1], for example, to change the value of the propagation constant in a line or the bandwidth of a microstrip line. Metal-dielectric structures can be placed near the radiating slot in the waveguide [2]. This metal-dielectric structure simulates the behavior of a closed diode, which is located near the emitting slot. Multilayer dielectric structures are placed in the laser aperture for mode selection [3]. Metaldielectric layers in a photonic nanostructure can act as a wide-aperture omnidirectional insulator [4]. A hybrid metal-dielectric nano-aperture antenna can be used to enhance surface fluorescence [5].

Modern telecommunication systems use microwaves in the millimeter and terahertz range. Antenna arrays are used as radiators in many telecommunication systems [6-11]. As a rule, antenna arrays are built on the basis of microstrip structures, open ends of waveguides, and horns. Recently, special interest has been shown to horn antenna arrays.

In [12], for the field description in the regions of the horn, the fractional index Hankel function expansion and the representation corresponding to the method of transverse numbers were used. The problem of excitation of field harmonics in horns is solved. Further, a system of linear differential equations was obtained for the amplitudes of field excitation in the horns.

Therefore, it is of interest to study the processes of radiation from an antenna array with a metal-dielectric structure and diffraction of a surface wave by a metal-dielectric structure.

\section{Problem formulation}

Considering the electromagnetic field domain (Fig. 1), one can see that the horns are not an extension of the waveguides. The horns are located at some distance from the waveguides in the radiation space of the antenna array (in the "Floquet channel").

Fig. 1 shows a unit cell of a linear infinite antenna array, which is located at the origin.

Let us conditionally divide the entire field domain in a single cell of an infinite antenna array into two regions:

Region 1 (waveguide): $-\infty \leq z \leq 0,-w / 2 \leq x \leq w / 2$.

Region 2 (spatial waveguide, "Floquet channel"): $0 \leq z \leq \infty,-f / 2 \leq x \leq f / 2$.

For area 2 , the recording of the horn in a single cell is performed based on the extended area method. 


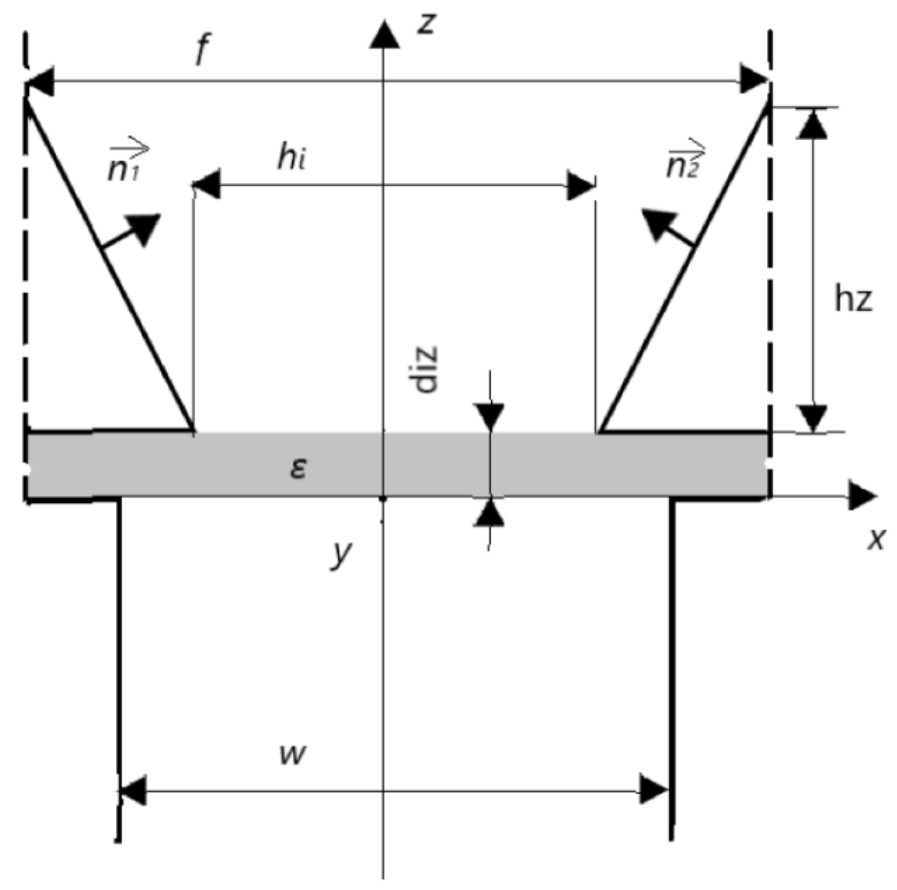

Fig. 1. Unit cell of linear infinite array.

Based on Green's second formula, the integral representation for the electric field strength of region 2 has the form:

$$
\begin{gathered}
E_{y}^{2}(x, z)=\int_{-w / 2}^{w / 2} E_{y}^{2 d}(x, 0) \frac{\partial G^{2 d}\left(x, z, x^{\prime}, z^{\prime}\right)}{\partial z^{\prime}} I_{z^{\prime}=0} d x^{\prime}+ \\
+\int_{-f / 2}^{-h i / 2 h z+d i z} \int_{d i z}\left[E_{y}^{2 h}\left(x^{\prime}, z^{\prime}\right) \frac{\partial G^{2 h}\left(x, z, x^{\prime}, z^{\prime}\right)}{\partial n_{1}}-G^{2 h}\left(x, z, x^{\prime}, z^{\prime}\right) \frac{\partial E_{y}^{2 h}\left(x^{\prime}, z^{\prime}\right)}{\partial n_{1}}\right] d z^{\prime} d x^{\prime}+ \\
+\int_{-f / 2}^{-h i / 2 h z+d i z}\left[E_{y}^{2 h}\left(x^{\prime}, z^{\prime}\right) \frac{\partial G^{2 h}\left(x, z, x^{\prime}, z^{\prime}\right)}{\partial n_{1}}-G^{2 h}\left(x, z, x^{\prime}, z^{\prime}\right) \frac{\partial E_{y}^{2 h}\left(x^{\prime}, z^{\prime}\right)}{\partial n_{1}}\right] d z^{\prime} d x^{\prime}+ \\
+\int_{h i / 2}^{f / 2 h z+d i z}\left[E_{y}^{2 h}\left(x^{\prime}, z^{\prime}\right) \frac{\partial G^{2 h}\left(x, z, x^{\prime}, z^{\prime}\right)}{\partial n_{2}}-G^{2 h}\left(x, z, x^{\prime}, z^{\prime}\right) \frac{\partial E_{y}^{2 h}\left(x^{\prime}, z^{\prime}\right)}{\partial n_{2}}\right] d z^{\prime} d x^{\prime}- \\
-\left.\int_{-f i / 2}^{2 d} G^{2 d}\left(x, z, x^{\prime}, z^{\prime}\right) \frac{\partial E_{y}^{2 d}\left(x^{\prime}, z^{\prime}\right)}{\partial z^{\prime}}\right|_{z^{\prime}=d i z} d x^{\prime}-\left.\int_{h i / 2}^{f / 2} G^{2 d}\left(x, z, x^{\prime}, z^{\prime}\right) \frac{\partial E_{y}^{2 d}\left(x^{\prime}, z^{\prime}\right)}{\partial z^{\prime}}\right|_{z^{\prime}=d i z} d x^{\prime} .
\end{gathered}
$$

The last two terms in the representation (1) 


$$
-\left.\int_{-f / 2}^{-h i / 2} G^{2 d}\left(x, z, x^{\prime}, z^{\prime}\right) \frac{\partial E_{y}^{2 d}\left(x^{\prime}, z^{\prime}\right)}{\partial z^{\prime}}\right|_{z^{\prime}=d i z} d x^{\prime}-\left.\int_{h i / 2}^{f / 2} G^{2 d}\left(x, z, x^{\prime}, z^{\prime}\right) \frac{\partial E_{y}^{2 d}\left(x^{\prime}, z^{\prime}\right)}{\partial z^{\prime}}\right|_{z^{\prime}=d i z} d x^{\prime}
$$

describe the case when there is only a resonator region with a dielectric in the field domain. The walls of this resonator region on one side are formed by waveguide flanges, on the other side by metal planes with dimensions $(f-h i) / 2$ along the $x$-axis. These metal planes in a unit cell are located symmetrically about the $z$-axis. Such a resonator region contains a dielectric with a dielectric constant $\varepsilon$.

We represent the electric field strength in region 2 as:

$$
\begin{gathered}
E_{y}^{2}(x, z)=\sum_{m f=-\infty}^{\infty}\left\{R D_{m f} f x_{m f}(x) e^{j \Gamma d(z-d i z)}+T D_{m f} f x_{m f}(x) e^{j \Gamma d(z-d i z)}\right\} \mathbb{I}_{0 \leq z \leq d i z} \\
E_{y}^{2}(x, z)=\left.\sum_{m f=-\infty}^{\infty}\left\{T H_{m f} f x_{m f}(x) e^{j \Gamma h(z-d i z)}\right\}\right|_{d i z \leq z \leq \infty}
\end{gathered}
$$

Here $R D_{m f}$ is the complex reflection coefficient of Floquet harmonics $(m f)$ in the region filled with a dielectric with a dielectric constant $\varepsilon ; T D_{m f}$ and $T H_{m f}$ are the complex coefficients of transmission of the Floquet harmonics ( $\mathrm{mf}$ ) in the region filled with a dielectric and in the region of the horn; $f x_{m f}$ is the complex transverse function of the "Floquet channel" [13]; $\Gamma d$ and $\Gamma h$ are longitudinal waveguide functions for the region that is filled with a dielectric and for the "Floquet channel" in the horn region [13]; $G^{2 d}$ and $G^{2 h}$ are Green's functions of region 2, which take into account the presence of several dielectric layers in the electromagnetic field domain [14].

It should be noted that in expression (2) the term

$$
R D_{m f} f x_{m f}(x) e^{j \Gamma d(z-d i z)}+T D_{m f} f x_{m f}(x) e^{j \Gamma d(z-d i z)}
$$

corresponds to the field component $E_{y}^{2 d}$ when the variable $z$ is changed within $0 \leq z \leq d i z$. The third term in expression (2) $T H_{m f} f x_{m f}(x) e^{j \Gamma h(z-d i z)}$ corresponds to the field component $E_{y}^{2 h}$ when the variable $z$ is changed within $\operatorname{diz} \leq z \leq \infty$.

The Green's function of the second region has the form:

$$
\begin{aligned}
& G^{2}\left(x, z, x^{\prime}, z^{\prime}\right)=\sum_{m f=-\infty}^{\infty} f x_{m f}(x) f x_{m f}^{*}(x) \frac{e^{\left.-j \Gamma d_{m g} \mid z^{\prime}-z\right]}}{2 j \Gamma d_{m q}}-2 k_{m g} \operatorname{sh}\left(j \Gamma d_{m d} z\right) \\
& \operatorname{sh}\left(j \Gamma d_{m d} z^{\prime}\right)-\frac{e^{-j \Gamma d_{m g}\left(z^{\prime}+z\right)}}{2 j \Gamma d_{m q}}, 0 \leq z \leq d i z \text {, for } G^{2 d}\left(x, z, x^{\prime}, z^{\prime}\right) ; \\
& G^{2}\left(x, z, x^{*}, z^{*}\right)=\sum_{m f=-\infty}^{\infty} f x_{m f}(x) f x_{m f}^{*}(x)\left(\frac{e^{-f\left(T \omega_{m g}-\Gamma d_{m g}\right) d i z}}{j \Gamma d_{m g}}+\right. \\
& \left.k_{m g}\left(e^{-j\left(\Gamma h_{m g}-\Gamma d_{m g}\right) d i z}-e^{-j\left(\Gamma h_{m g}+\Gamma d_{m g}\right) d i z}\right)\right) \times \\
& e^{-j \Gamma h_{m g} z^{2}} \operatorname{sh}\left(j \Gamma d_{m d} z\right), d i z \leq z \leq \infty \text {, for } G^{2 h}\left(x, z, x^{\prime}, z^{\prime}\right) \text {. }
\end{aligned}
$$


The notation is introduced here:

$$
k_{m g}=\frac{\left(j \Gamma h_{m g}-j \Gamma d_{m g}\right) e^{-2 j \Gamma d_{m g} d i z}}{j r d_{m g}\left[j \Gamma h_{m g}+j \Gamma d_{m g}-j\left(\Gamma_{h g}-\Gamma d_{m g}\right) e^{-1 j \Gamma d_{m g} d i z}\right]} .
$$

We will assume that the expression for the electric field strength in region 1 has the form:

$$
E_{y}^{1}(x, z)=E_{e x c}(x, z)+\sum_{m w=1}^{\infty} R_{m w} W X_{m w}(x) e^{j \gamma_{m w} z}
$$

Here $E_{\text {exc }}(x, z)=W X_{1} e^{j Y_{1} z}+\sum_{m w=1}^{\infty} R_{m w} W X_{m w} e^{j Y_{m w} z}$ is the electric field intensity of the incident wave $H_{10}$ in the waveguide; $R_{m w}$ is a complex mode mw reflection coefficient; $W X_{m w}(x)$ are transverse eigenfunctions of the waveguide for the wave $H_{m w 0} ; \gamma_{m w}$ is a longitudinal propagation coefficient of the waveguide mode $m w$.

We use the boundary conditions for the tangential components of electric field strength vectors and their derivatives along the normal at $z=0$ :

$$
\begin{gathered}
E_{y}^{2}(x, 0)=E_{y}^{1}(x, 0) \\
\frac{\partial E_{y}^{2}(x, z)}{\partial z} \mathrm{I}_{z=0}=\frac{\partial E_{y}^{1}(x, z)}{\partial z} \mathrm{I}_{z=0} .
\end{gathered}
$$

We also use the boundary conditions for the electric field strength of region 2 at $z=d i z$ :

$$
\begin{gathered}
E_{y}^{2 d}(x, d i z)=E_{y}^{1 / h}(x, d i z) ; \\
\left.\frac{\partial E_{y}^{2 d}(x, z)}{\partial z}\right|_{z=d i z}=\left.\frac{\partial E_{y}^{1 / f}(x, z)}{\partial z}\right|_{z=d i z^{*}}
\end{gathered}
$$

The use of the orthogonality condition for the transverse eigenfunctions makes it possible to exclude the dependence on the coordinate $x$ from the obtained equations. As a result of some transformations, a system of linear algebraic equations for complex amplitude coefficients $R D_{m f}, T D_{m f}, T H_{m f}, R_{m w}$ is obtained.

\section{Conclusions}

Electrodynamical calculation of an infinite waveguide antenna array, which contains a metal-dielectric structure in the near zone of the antenna array, is carried out by the method of the integral equation. The metal-dielectric structure is a combination of a dielectric coating over the antenna array and horns. The horns are not connected to the waveguides and are located at some distance from the antenna array.

A system of equations that allows calculating the reflection coefficients of incident waves in waveguides is obtained. 


\section{References}

1. Vahidek, R. Accurate Hybrid-Mode Finline Configurations Including Analysis of Various Multilayered Dielectrics, Finite Metallization Thickness, and Substrate Holding Grooves // IEEE Transactions on Microwave Theory and Techniques. - 1984. - Vol. 32. - No. 11. - P. $1454-1460$.

2. Kryzhanovskiy, V.G. Analiz volnovodno-shchelevoy linii peredachi s pryamougol'noy metallo-dielektricheskoy vstavkoy v shcheli / K.G. Kryzhanovskiy, Yu.V. Rasokhina // Izvestiya Vysshikh Uchebnykh Zavedeniy. Radioelektronika. - 2008. -No. 5. - P. $72-78$.

3. Ilchenko, S.G. Multilayer dielectric structure for mode selection of wide-aperture laser / S.G. Ilchenko, R.A. Lymarenko, V. Naranenko // Proceedings of IEEE 8th International Conference on Advanced Optoelectronics and Lasers (CAOL'2019) - 2019. - Mode access: https://ieeexplore.ieee.org/document/9019492

4. Chabanov, A.A. Metal-dielectric photonic structures with extreme directionality: A concept of wide-aperture omnidirectional isolator / A.A. Chabanov, K. Smith, T. Carroll, I. Vitebskiy // Proceedings of IEEE 8th International Congress on Advanced Electromagnetic Materials in Microwaves and Optics. - 2014. - Mode access: https://ieeexplore.ieee.org/document/6948601

5. Lu, G. Hybrid metal-dielectric nano-aperture antenna for surface enhanced fluorescence / G. Lu, J. Xu, T. Wen, W. Zhang // Materials. - 2018. - Vol. 11. - No. 8. P. $1435-1438$.

6. Rey, S. A phased array antenna with horn elements for $300 \mathrm{GHz}$ communications / S. Rey, T. Merkle, A. Tessmann, T. Kurner // Proceedings of IEEE International Symposium on Antennas and Propagation (ISAP'2016). - 2016. - Mode access: https://ieeexplore.ieee.org/document/7821108

7. Schulwitz, L. A compact millimeter-wave horn antenna array fabricated through layer-by-layer stereolithography / L. Schulwitz, A. Mortazawi // Proceedings of IEEE Antennas and Propagation Society International Symposium. - 2008. - Mode access: https://ieeexplore.ieee.org/document/4619409

8. Peng, M. High performance $5 \mathrm{G}$ millimeter-wave antenna array for $37-40 \mathrm{GHz}$ mobile application / M. Peng, A. Zhao // Proceedings of IEEE International Workshop on Antenna Technology (IWAT'2018). - 2018. - Mode access: https://ieeexplore.ieee.org/document/8379148

9. Feshawey, M.H. Comparison between 23 D-Printed $2 \times 2$ Horn Antenna Arrays at $12.5 \mathrm{GHz}$ with Different Feeding Structures / M.H. Feshawey, H.F. Hammad // Proceedings of IEEE International RF and Microwave Conference (RFM'2018). - 2018. - Mode access: https://ieeexplore.ieee.org/document/8846541

10. Wang, J. Four-element TEM horn array for radiating ultrawideband electromagnetic pulses / J. Wang, C. Tian, G. Luo, Y. Chen, D. Ge // Microwave and Optical Technology Letters. - 2001. - Vol. 31. - No. 3. - P. 190 - 194.

11. Kaloshin, V.A. Issledovaniye kharakteristik Ye-ploskostnykh lineynykh reshetok TEM ruporov / V.A. Kaloshin, K.Z. Nguyen // Zhurnal radioelektroniki. - 2017. - No. 5. - Mode access: http://jre.cplire.ru/jre/may17/14/text.pdf

12. Aleksandrov, N.L. Kharakteristiki izlucheniya reshetki ruporov / N.L. Aleksandrov, Yu.P. Vinichenko, A.A. Lemanskiy // Radiotekhnika i elektronika. - 1986. - No. 6. - P. 1026 - 1032.

13. Amitay, N. Theory and analysis of phased array antennas / N. Amitay, V. Galindo, C. Wu. - New York: Wiley-Interscience, 1972. - 462 p. 
14. Prokhoda, I.G. Tenzornye funktsii Grina i ikh primeneniye v electrodinamike SVCh / I.G. Prokhoda, S.G. Dmitriuk, V.M. Morozov. - Dneproperovsk: DGU, 1985. $64 \mathrm{p}$. 